\title{
The Feasibility of Conventional Beamforming Algorithm Based on Resolution for Internet of Things in Millimeter Wave Environment
}

\author{
Yuehong Guo, Lixin Li, Xiaocong Wen, Ang Gao, Yatai Liu \\ School of Electronics and Information, \\ Northwestern Polytechnical University, Xi'an, Shaanxi 710129, China \\ E-mail:guoyuehong@mail.nwpu.edu.cn
}

\begin{abstract}
With the densification of Internet of Things (IoT), the IoT application will benefit a lot from a wide range of unused bandwidths Millimeter wave (mm wave) frequencies. Through the general theoretical background, this paper puts forward several conventional beamforming algorithms in the mm wave environment, and analyzes the feasibility of these algorithms based on resolution by simulation. It is found that considering the position deviation of the virtual antenna array in M2M communication, the main lobe of the CBF, MVDR, LMS, MUSIC algorithm obtains the maximum value in the DOA, while the beam pattern of the CBF, MVDR, LMS algorithms have a large deviation from the directions except DOA. The beam pattern of MUSIC algorithm is almost the same as the expected response of the uniform array. The simulation results show that MUSIC algorithm can be used in the mm wave environment of high-performance IoT communication.
\end{abstract}

Keywords: Millimeter wave; Beamforming; IoT

\section{Introduction}

The Peak data rates, network capacity, and coverage areas are steadily increasing, mobile communication data traffic showed an exponential growth trend. The spectrum resources of microwave under $3 \mathrm{GHz}$ is increasingly scarce, it will eventually be unable to meet the growing high-speed transmission needs. Mm wave frequency band $30-300 \mathrm{GHz}$ has a very rich spectrum resources, the development of mm wave broadband wireless communications has strategic significance. The short wavelength of $\mathrm{mm}$ wave can realize the integration of antenna arrays in $\mathrm{mm}$ wave communication system to facilitate the hot spot area-intensive base station layout. However, due to its short wavelength will bring serious path loss, beamforming becomes a compensation path loss and strategies for improving transmission quality [1].

The coming service and application environment for the next decade will include smart cities and related applications, wearable computing, smart and telemedicine and education, and security and lifeline systems. The IoT will be the foundation for building these services [2]. At the end of 2014, there were 2.9 billion Internet users and 7.4 billion mobile connections. The number of M2M connections is expected to increase to 3.2 billion By 2019, reaching a compound annual growth rate of 45\% [3]. The network capacity and throughput requirements from beyond Four-Generation (B4G) mobile systems are difficult to achieve in the highly congested 6-GHz band. The addition of billions of new M2M links is also unbearable. However, densification of the device results in a shorter transmission distance, thus creating a suitable environment for the use of higher frequency bands, while greater path loss is traditionally used to prevent general communication. The IoT application will benefit from a wide range of unused bandwidths $\mathrm{mm}$ wave frequencies, extending from $30 \mathrm{GHz}$ to $300 \mathrm{GHz}$. Mm wave communications system has clear vision to be used in the environment of IoT [4-6].

Many nodes in the IoT cooperate to form a large antenna array, thus greatly improving the transmission range and data rate. Beamforming is the process of combining signals from different elements to form a single output of the sensor array. Many studies have shown that when the antenna array is properly configured, it can both improve channel capacity and extend coverage, as well as reduce multipath fading and bit error rate (BER). The antenna gain is proportional to the number of elements, so the peak power density of the main beam can be several orders of magnitude higher than a single sensor. Another useful feature of an antenna array is that it can perform temporal filtering, suppressing potentially interfering signals in the directions except the desired directions. This feature can be easily implemented using zero techniques [7]. In summary, in view of these advantages, beamforming can achieve the goal of building an efficient IoT.

Some beamforming algorithms have been successfully applied in traditional antenna arrays. However, these algorithms that calculate the weights of the array elements cannot be directly applied to IoT because of significant differences between the IoT and conventional arrays. For example, a phased array in a radar is permanently mounted at a fixed location, i.e., the position of the element is fixed. However, nodes in IoT are 
usually deployed at random, and their relative positions are not predetermined. Since this random configuration has a position deviation compared to an equidistant array, this will degrade the performance of the array.

This paper puts forward several conventional beamforming algorithms in the $\mathrm{mm}$ wave environment, and analyzes the feasibility of these algorithms based on resolution by simulation. It is found that considering the position deviation of the virtual antenna array in M2M communication, the main lobe of the CBF, MVDR, LMS, MUSIC algorithm obtains the maximum value in the DOA, while the beam pattern of the CBF, MVDR, LMS algorithms have a large deviation from the directions except DOA. The beam pattern of MUSIC algorithm is almost the same as the expected response of the uniform array. The simulation results show that MUSIC algorithm can be used in the mm wave environment of high-performance IoT communication.

\section{Beam pattern simulation}

\subsection{Model establishment}

With the development of micro-electro-mechanical systems (MEMS) technology, it is becoming easier to manufacture sensors which have high signal processing and communication capabilities, and they are small in size and cheap. These sensor nodes are deployed in the location of interest and are used to collect, process and transmit information. However, the implementation of an effective sensor network still faces many challenges, such as node power consumption, node controllable deployment, resource location, self-organization and so on.

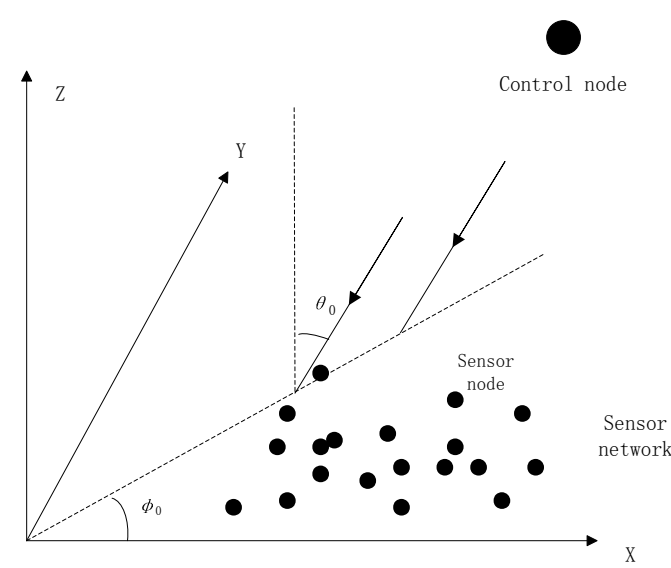

Figure 1. The diagram of the IoT

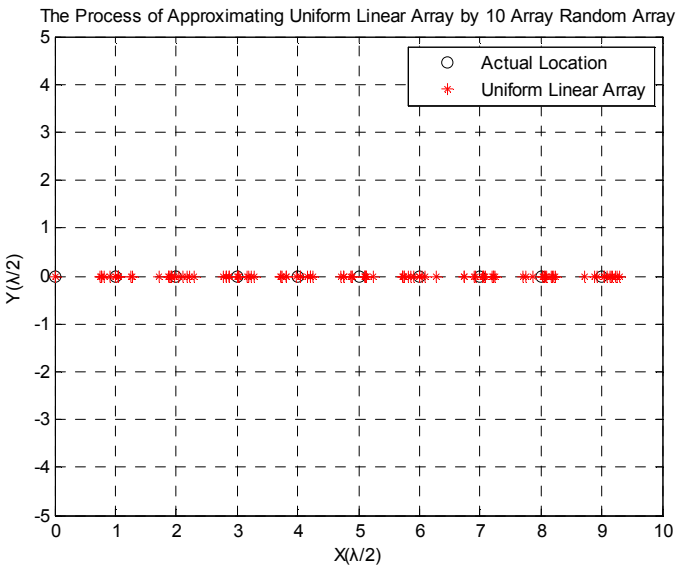

Figure 2. The process of a series of nodes approximate the uniform linear array

The application scenario depicted in Figure 1 is used in this paper. The space Cartesian coordinate system Oxyz represents a three-dimensional space. A set of sensor nodes are deployed at locations of interest to obtain the desired information, which may be any representation of the signal (sound, video, etc.). The control node establishes a connection with the sensor network above the sensor area to obtain the collected data. According to some geometric criteria, a central node (cluster head) tries to find a set of nodes whose topology is uniform linear or planar array. On the premise that only the distance between nodes is available, some algorithms try to find the optimal sensor subset which minimizes the average position deviation [8]. In Figure $1, \theta_{0}$ and $\varphi_{0}$ are the elevation and azimuth of the transmitted signals of the control node, respectively, which subject to $0^{\circ} \leq \theta_{0} \leq 90^{\circ}, 0 \leq \phi_{0} \leq 360^{\circ}$. Figure 2 illustrates this process, in which a series of sensor nodes approximately forms a uniform linear array with an interval of $\lambda / 2$, and $\lambda$ is the incident signal wavelength. After finding the best set of nodes, some beamforming schemes are applied to obtain the weighting coefficients.

\subsection{Beamforming algorithms}

It is well-known that the basic beamforming algorithms include cbf, mvdr, lms, rls, music, etc., and there are many improved algorithms based on these algorithms. This paper focuses on these four basic algorithms, CBF, MVDR, LMS and MUSIC. The CBF is simple to implement, but it has low angular resolution. MVDR is a classic beamforming algorithm, and it can also be used to estimate DOA, but its resolution is relatively low. The calculation of LMS algorithm is small, but it requires training sequences, and convergence is slow. MUSIC algorithm has a universal applicability and a high resolution, but it cannot distinguish the strong correlation signal. Next, we introduce the implementation of these algorithms, considering the impact of the Internet of Things in mm wave environment. 


\subsubsection{CBF algorithm}

The beamformer is an extension of the conventional beamformer, but it takes into account random position deviation. In this method, for a uniform linear array, the weight $\vec{w}$ is selected to match the steering vector $\overrightarrow{a\left(\theta_{0}\right)}$, and the maximum gain of the main lobe is obtained in the direction of the DOA $\theta_{0}$. If the steering vector

$$
\overrightarrow{a\left(\theta_{0}\right)}=\left[\begin{array}{lllll}
1 & e^{-j 2 \pi x_{2} / \lambda \sin \theta_{0}} & e^{-j 2 \pi x_{3} / \lambda \sin \theta_{0}} & \ldots & e^{-j 2 \pi x_{n} / \lambda \sin \theta_{0}}
\end{array}\right]^{T}
$$

The received signal is $X=\left[\begin{array}{llll}x_{1}(t) & x_{2}(t) & \ldots & x_{n}(t)\end{array}\right]^{T}$, where $(\square)^{T}$ represents the transpose of the vector $(\square)$, $n$ denotes the number of elements.

Then $\vec{w}=\overrightarrow{a\left(\theta_{0}\right)}$. The amplitude of each element of the weight vector is 1 , and the phase is the same as the corresponding element of the steering vector. As a result, the array responds

$$
F_{C B F}(\theta)=\vec{w}^{H} R \overrightarrow{a(\theta)}
$$

The covariance matrix $R=E\left[X X^{H}\right]$, where $(\square)^{H}$ represents the conjugate transpose of the vector $(\square)$, and the maximum value of $F_{C B F}(\theta)$ is in the DOA $\theta_{0}$.

The distance $x 1, x 2, \ldots, x n$ between the element and the reference node is not a multiple of the ideal distance $\lambda / 2$ due to the position deviation in the sensor array. However, the selected weight vector can still be matched to the steering vector in the DOA $\theta_{0}$. In the sensor network, this implementation is very simple, in order to simplify the problem, make the following assumptions:

1) Each node can accurately calculate the distance from the reference node location.

2) For the hierarchical structure of the cluster, the relative position of the node and the cluster head is decentralized.

3) The desired direction of the input signal to the central node is known.

These assumptions also apply to other algorithms.

\subsubsection{MVDR algorithm}

MVDR is an adaptive beamforming algorithm based on the maximum signal-to-interference-to-noise ratio (SINR) criterion. In this method, the steering vector $\overrightarrow{a\left(\theta_{0}\right)}$, the received signal $X$, covariance matrix $R$ are the same as the above. The weight vector

$$
\vec{w}=\mu R^{-1} \overrightarrow{a\left(\theta_{0}\right)}
$$

Where $\mu=\frac{1}{\overline{a\left(\theta_{0}\right)} R^{-1} \overline{a\left(\theta_{0}\right)}},(\square)^{-1}$ represents the inverse of the matrix $(\square)$. As a result, the array responds is

$$
F_{M V D R}(\theta)=\vec{w}^{H} \overrightarrow{a(\theta)}
$$

And the maximum value of $F_{M V D R}(\theta)$ is in the DOA $\theta_{0}$.

\subsubsection{LMS algorithm}

LMS algorithm has the characteristics of low computational complexity and good convergence, and is the most widely used adaptive algorithm. In this method, the steering vector $\overrightarrow{a\left(\theta_{0}\right)}$, the received signal $X$, covariance matrix $R$ are the same as the above. The expected response is $\overrightarrow{d(k)}$. The iterative formula of weight vector is

$$
\overrightarrow{w(k+1)}=\overrightarrow{w(k)}+\mu \overrightarrow{x(k)} \overrightarrow{e(k)}
$$

Where $\mu$ is the step size parameter, which subject to $0<\mu<\operatorname{tr}(R)$, where $\operatorname{tr}(\square)$ represents the trace of the matrix $(\square) . \overrightarrow{e(k)}$ represents the instantaneous error between the array output and the expected response $\overrightarrow{d(k)}$.

$$
\overrightarrow{e(k)}=\overrightarrow{d(k)}-\overrightarrow{w(k)}^{H} \overrightarrow{x(k)}
$$

And the array responds can be expressed as

$$
F_{L M S}(\theta)=\vec{w}^{H} \overrightarrow{a(\theta)}
$$

\subsubsection{MUSIC algorithm}

The MUSIC algorithm is a method based on matrix feature space decomposition. The observation space of signal processing is decomposed into signal subspace $U_{S}$ and noise subspace $U_{N}$ by the following equation

$$
R=U_{S} \Sigma_{S} U_{S}{ }^{H}+U_{N} \Sigma_{N} U_{N}{ }^{H}
$$


After the eigen-decomposition of $R, k$ larger eigenvalues constitute diagonal matrix $\Sigma_{S}$, the corresponding eigenvectors constitute the signal subspace $U_{S}, k$ is the number of signals, the rest eigenvalues constitute diagonal matrix $U_{N}$, and the corresponding eigenvectors constitute the signal subspace $U_{N}$.

And it is clear that these two spaces are orthogonal. The MUSIC algorithm utilizes the orthogonality between the two complementary spaces to estimate the azimuth of the spatial signal. MUSIC algorithm greatly improves the direction of the resolution, while adapting to any shape of the antenna array. The array responds can be expressed as

$$
F_{\text {MUSIC }}(\theta)=\frac{1}{\overrightarrow{a(\theta)}{ }^{H} U_{N} U_{N}{ }^{H} \overrightarrow{a(\theta)}}
$$

\section{Simulation and results}

The array element spacing is generally set to $\lambda / 2$ in the uniform linear array, so the position deviation is set to be $0.4 \lambda / 2$ in the simulations, which is large enough and the actual position of the sensor node is closer to the corresponding node in the uniform linear array. The DOA of the expected signal is $\theta_{0}=0^{\circ}$. The graph is the average beam pattern after 50 times simulation. Figure 3, Figure 4 and Figure 5 show that, when using CBF, MVDR and LMS algorithms, the main lobes of the beam patterns in the DOA $\theta_{0}$ get the maximum, the same as the maximum value of uniform linear array (ideal). However, the beam pattern indicates a large deviation from the other directions. That is, the performance of the array approaches the uniform linear array in the vicinity of the DOA direction, but the performance degrades significantly in other directions.

Figure 6 shows the main lobe of the beam pattern in the DOA $\theta_{0}$ get the maximum. The beam pattern of MUSIC algorithm indicates a slight deviation from other directions. That is, the performance of the array approaches the uniform linear array in the vicinity of the DOA, and the performance degrades a little in other directions.

Figure 7 compares different beamforming algorithms for a $10 \times 1$ linear array with a position deviation of $0.4 \lambda / 2$. Because CBF algorithm is ideal, so just three algorithms, MVDR, LMS and MUSIC, are compared. It is clear to see from the figure that the MUSIC algorithm has the best resolution when there is a position deviation, followed by the MVDR algorithm, and the LMS algorithm suffers the biggest performance loss. The reason is that, using MUSIC algorithm to determine the weight can indeed eliminate the impact of position deviation.

In summary, MUSIC algorithm is a good choice when we put the IoT into the $\mathrm{mm}$ wave environment to realize high-performance communication using beamforming.

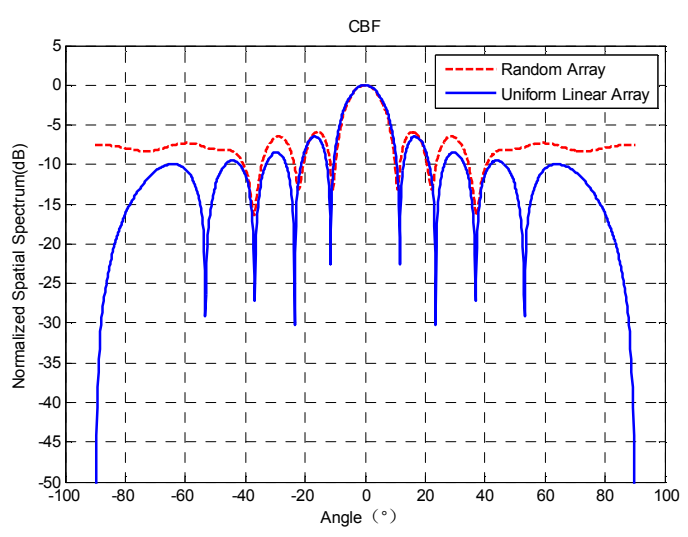

Figure 3. Comparison of beam patterns for a $10 \times 1$ linear array with position deviation and $10 \times 1$ uniform linear array using $\mathrm{CBF}$ algorithm

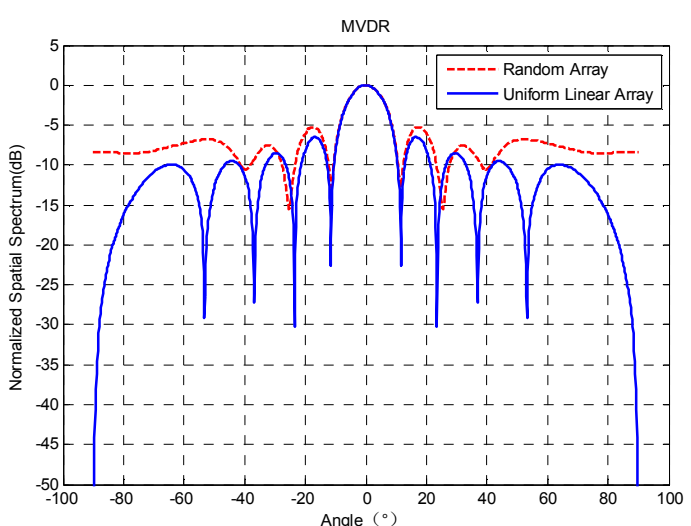

Figure 4. Comparison of beam patterns for a $10 \times 1$ linear array with position deviation and $10 \times 1$ uniform linear array using MVDR algorithm 


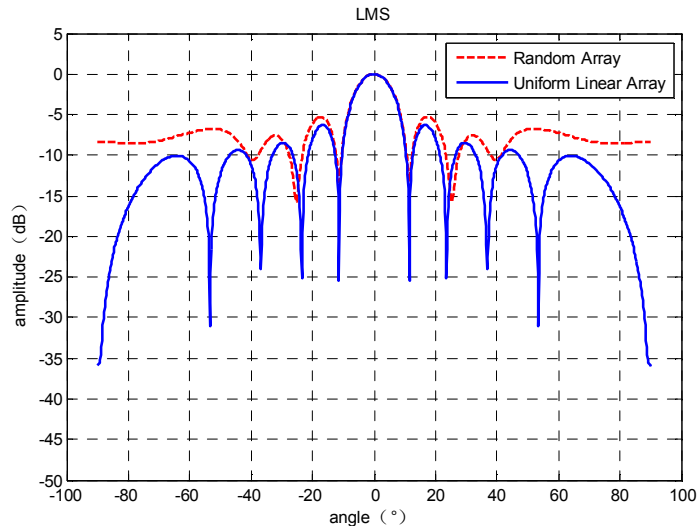

Figure 5. Comparison of beam patterns for a $10 \times 1$ linear array with position deviation and $10 \times 1$ uniform linear array using LMS algorithm

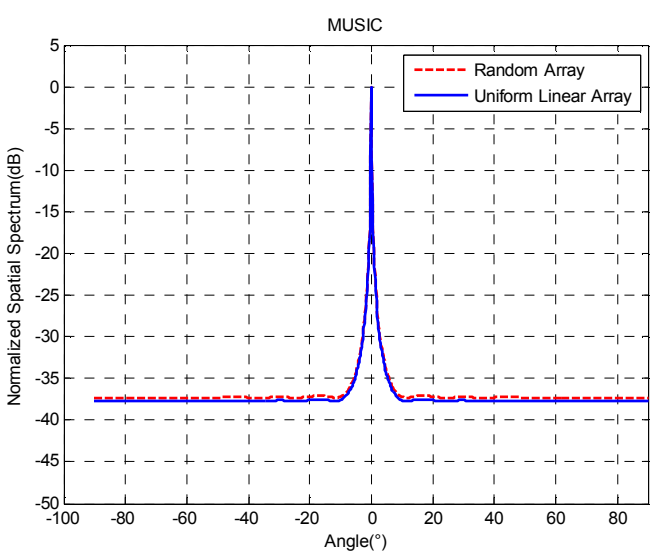

Figure 6. Comparison of beam patterns for a $10 \times 1$ linear array with position deviation and $10 \times 1$ uniform linear array using MUSIC algorithm

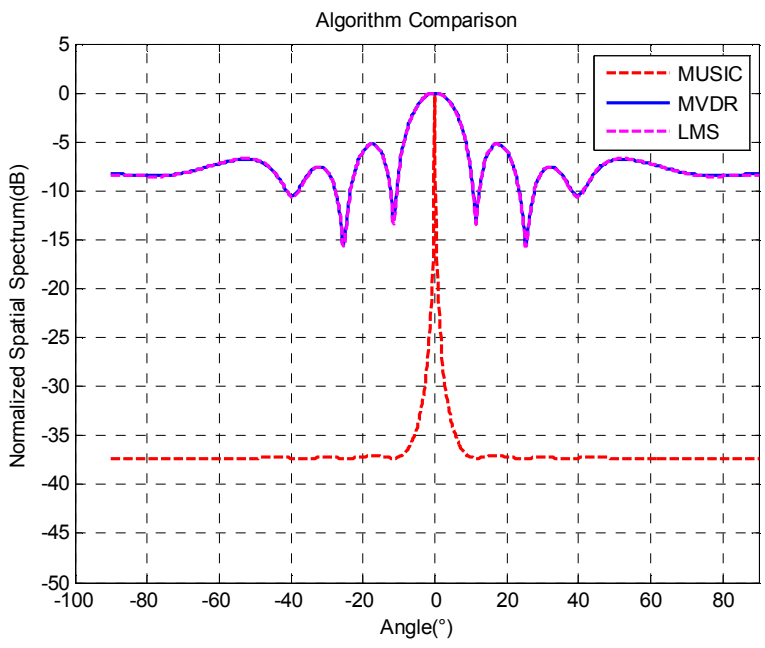

Figure 7. Comparison of different beamforming algorithms for a $10 \times 1$ linear array with a position deviation of $0.4 \lambda / 2$

\section{Conclusion}

According to the general theoretical background of the IoT, this paper presents several conventional beamforming algorithms in the mm wave environment, and analyzes the feasibility of these algorithms based on resolution by simulation. Because the beam pattern of the CBF, MVDR and LMS algorithms have a large deviation from directions except DOA, so they are no longer applicable in the mm wave environment for the IoT. The MUSIC algorithm achieves almost the same response of the uniform linear array, so it can be used as the basic beamforming algorithm in the $\mathrm{mm}$ wave environment.

In traditional arrays, beamforming is performed by a single processor, and all information is collected in a central processing node responsible for solving the beamforming problem. In the IoT environment, because a single node takes on the whole arduous computing task, if the node fails, the beamforming problem must be solved once more. Therefore, by improving the algorithm to effectively distribute the processing burden in the entire IoT to reduce the average power consumption of nodes and improve the robustness of the network, further research is needed. 


\section{Acknowledgement}

This work is supported by Shaanxi Province natural science basic research plan surface project (No. 2016JM6062), by China Aerospace Science and Technology Corporation Aerospace Science and Technology Innovation Fund funded project, by the Seed Foundation of Innovation and Creation for Graduate Students in Northwestern Polytechnical University.

\section{References}

[1] Kutty S, Sen D. Beamforming for Millimeter Wave Communications: An Inclusive Survey. IEEE Communications Surveys \& Tutorials, 2016: 949-973.

[2] V. Gazis et al. Short Paper: IoT: Challenges, projects, architectures. 2015 18th International Conference on Intelligence in Next Generation Networks (ICIN). IEEE. 2015:145-147.

[3] Cisco. Cisco visual networking index: Global mobile data traffic forecast update, 20102015. Whitepaper, Feb. 2011.

[4] S. Borkar and H. Pande. Application of 5G next generation network to Internet of Things. 2016 International Conference on Internet of Things and Applications (IOTA). IEEE. 2016:443-447.

[5] Yilmaz T, Gokkoca G, Akan O B. Millimetre Wave Communication for 5G IoT Applications. Internet of Things (IoT) in 5G Mobile Technologies. Springer International Publishing, 2016: 37-53.

[6] Jornet J M, Akyildiz I F. Channel Modeling and Capacity Analysis for Electromagnetic Wireless Nanonetworks in the Terahertz Band. IEEE Transactions on Wireless Communications, 2011:3211-3221.

[7] Saponara S, Neri B. mm-wave integrated wireless transceivers: Enabling technology for high bandwidth connections in IoT. 2015 IEEE 2nd World Forum on Internet of Things (WF-IoT). IEEE. 2015:149-153.

[8] Mora F C, Xiao J. Preprocessing technique to signal strengthdata of wireless sensor network for real-time distance estimation. 2008 IEEE International Conference on Robotics and Automation. IEEE. 2008: 1537-1542. 